\title{
Enhancement of Mode Stability in Vertical Cavity Surface Emitting Lasers with Diffused Quantum Wells Structure
}

\author{
E.Herbert Li \& S.F. Yu ${ }^{+}$ \\ Department of Electrical and Electronic Engineering, University of Hong Kong, Pokfulam Road, H.K. \\ Tel: +852-2859-8026, Fax: +852-2559-8738, ${ }^{+}$Email: sfyu@hkueee.hku.hk
}

Abstract - The use of diffused quantum well structures to enhance single transverse mode operation in vertical cavity surface emitting semiconductor lasers is proposed and analyzed theoretically.

Vertical cavity surface emitting lasers (VCSEL's) show promising characteristics for various applications such as high speed parallel optical communication and computing systems. However, VCSEL's exhibit multiple transverse modes operation at high power which deteriorate the performance of optical communication systems. The reasons for multi-mode operation are due to 1) spatial hole burning of carrier concentration and 2) thermal lensing effect.

In this paper, a step diffused quantum wells (DFQW's) structure is proposed to overcome the influence of spatial hole burning and thermal lensing in VCSEL's [1,2]. Step DFQW's structure can be realized by using a circular hollow mask to define the DFQW's structure, see figure 1 .

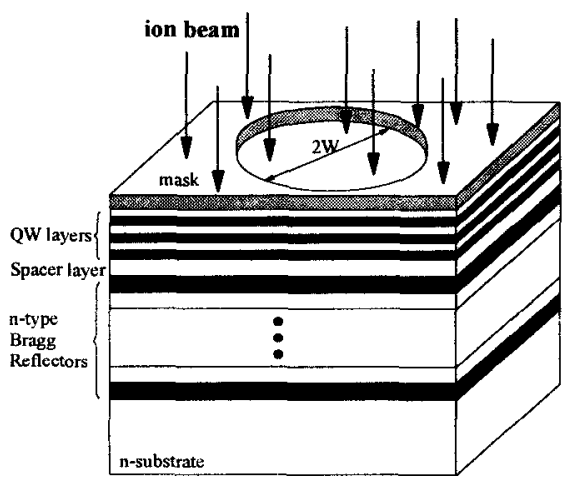

Fig 1 Implantation on QW layers of VCSEL's.

In our analysis, we assumed the laser has a circular metal contact of diameter $10 \mu \mathrm{m}$ on the expitaxial side ( $\mathrm{p}$-side) for current injection. The GaAs- $\mathrm{Al}_{0.3} \mathrm{Ga}_{0.7} \mathrm{As}$ quantum well active layer is sandwiched between two undoped spacer layers and two Bragg reflectors. The undoped spacer layers have thickness of half-wavelength each. The Bragg reflectors are formed by alternating layers of $\mathrm{AlAs}$ and $\mathrm{AlGaAs}$ with quarterwavelength thickness dielectric layers on both the $\mathrm{n}$ - and $\mathrm{p}$-side, respectively. The total number of layers is equal to 26 on the n-side and 68 on the p-side. The active region consists of six $\mathrm{GaAs}-\mathrm{Al}_{0.3} \mathrm{Ga}_{0.7}$ As quantum wells with well width of $100 \AA$ and the total thickness of the active layer is half-wavelength.

The multi-mode operation in gain guided VCSEL's is caused by the increase in refractive index inside the core region (due to spatial hole burning and thermal lensing effects) such that transverse modes are collimated into the center of core region. Figure 2 shows the profile of fundamental $\left(\mathrm{LP}_{01}\right)$ and first order $\left(\mathrm{LP}_{11}\right)$ modes at and twice its threshold bias.

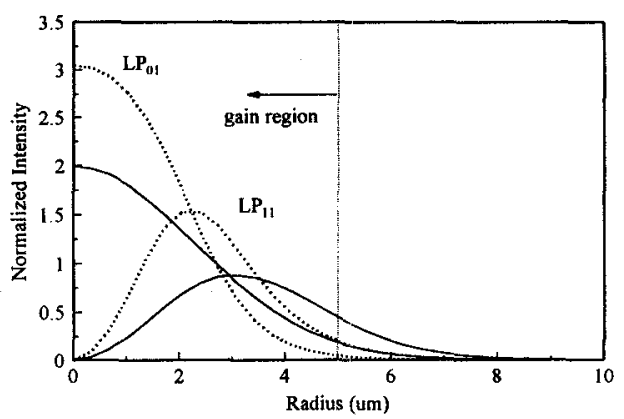

Figure 2 Transverse modes distribution of VCSEL's at threshold (solid line) and twice its threshold bias (dotted line).

As we can see, transverse modes are moving toward the center of core. As a result, the effective optical gain of the $L_{11}$ is enhanced due to the increase in overlapping integral between gain (core) region and optical field. From the above phenomena, stable single mode operation of VCSEL's can be achieved 
provided that the refractive index profile of the core can be alternated. This can be done by compositional induced disordering into the active QW layer such that the refractive index is selectively reduced within the core region to form an anti-guiding structure for the transverse modes.

In our calculation, the extent of interdiffusion into the QW active layer is characterized by a diffusion length, $L_{d}$ (where $L_{d}=0 \AA$ represents the as-grown $\mathrm{QW})$. The corresponding parameters such as optical gain and refractive index of the DFQW's material can be obtained from [2] and are used for the following calculations. Furthermore, the transverse modes behavior of VCSEL's is investigated by using the recently developed laser model [3]. In addition, the 3-D distribution of voltage, temperature and impurities concentration are also introduced into the model such that the influence of spatial hole burning and thermal lensing can be taken into consideration.

Figure 3 shows the light/current curves for VCSEL's without (dotted line) and with (solid line) a step DFQW structure of $L_{d}=5 \AA$. The radius of the diffusion area, $w$, is set to 3 and $3.8 \mu \mathrm{m}$. In the calculation, it is found that at low output power only $\mathrm{LP}_{01}$ is dominated. However, $\mathrm{LP}_{11}$ is excited above the kink in the light/current curves. The kink can be shifted to a higher power level by using a step DFQW's structure with suitable diffusion area.

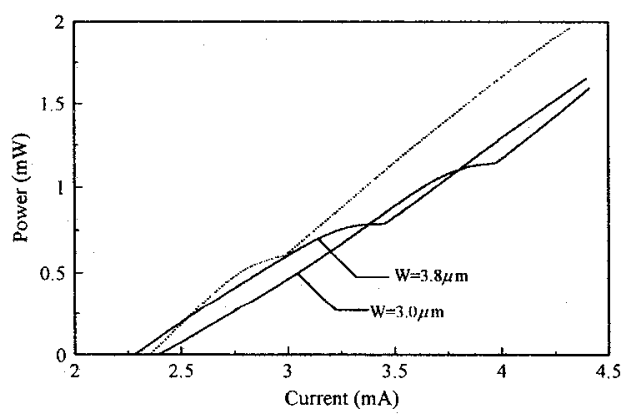

Figure 3 Light/current curves of VCSEL's without (dotted line) and with (solid line) a step DFQW structure.

Figure 4 shows the refractive index profile for both devices without (dotted line) and with (solid line) a step DFQW structure at output power of $1 \mathrm{~mW}$. The refractive index profile for VCSEL's without DFQW's structure at threshold is also shown in the figure for comparison. As we can see for the case without step DFQW's structure, the refractive index is increase near the center of the core. This move transverse modes towards the center region and the $\mathrm{LP}_{11}$ is excited. However, the anti-guiding structure of the step DFQW's structure counteracts the increase in refractive index and maintain the domination of $\mathrm{LP}_{01}$ mode.

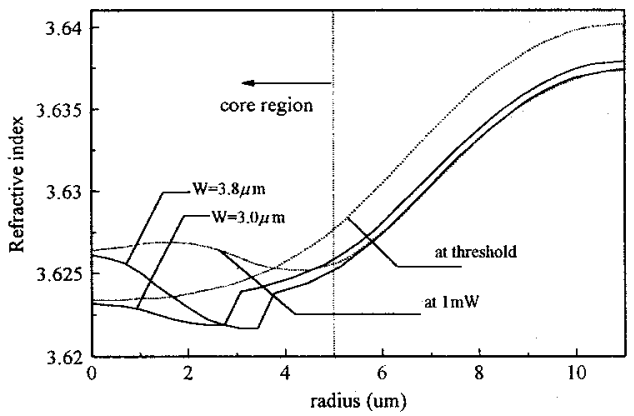

Figure 4 Refractive index distribution of VCSEL's without (dotted line) and with (solid line) a step DFQW structure.

In conclusion, we have shown that a step DFQW's structure can be utilized to enhance stable single mode operation in VCSEL's. It is found that the radius of DFQW's structure has significant influence on the performance of the light/current characteristics. Analysis and explanation on the enhancement of stable single mode operation by using DFQW's structure will be discussed in details in the presentation.

\section{References}

[1] S.F. Yu \& E.H. Li,' 'Proposed enhancement of side mode suppression ratio in $\lambda / 4$ shifted distributed feedback lasers with non-uniform diffused quantum wells', IEEE Photon. Technol. Lett., Vol. 8, no. 4, pp. 482-484, 1996.

[2] S.F. Yu, C.W. Lo \& E.H.Li,'Investigation of high power single mode operation in DFB and FP lasers using diffused quantum wells structure', IEEE J. Quantum Electron, Vol. 33, no.6, pp.999-1009, 1997.

[3] S.F. Yu,'Dynamic behavior of vertical cavity surface emitting lasers', IEEE J. Quantum Electron., Vol. 32, no.7, pp. 1168-1179, 1996. 